\title{
Reference Signal Processing for Aging State Recognition
}

\author{
Mikhail Olkhovskiy, Eva Müllerová, Petr Martínek \\ Department of Electrical Power Engineering and Environmental Engineering, \\ Faculty of electrical engeeniring, \\ University of West Bohemia, \\ Pilsen, Czech Republic \\ mikhail@fel.zcu.cz, mullerov@fel.zcu.cz,petrmart@fel.zcu.cz
}

\begin{abstract}
In this work we presented the results of the experiment in which a reference signal was used for determining degradation level of cable insulation. A set of reference signals was constructed using programing language. Onwards, each variant of the constructed signal was subsequently sent to the coaxial cable by aim of a following: digital-analog converter, radio frequency power amplifier with high linearity, and high frequency current injection air core transformer. The signals, passed through the cable, were received with a current transformer and processed using algorithm written in Python language. Processed signals were utilized for constructing data set for modern one-dimensional convolutional neural network. Neural network was defined in Keras, then optimal configuration of the network structure and its parameters were found. Also, visualization by gradient-based localization method was used to interpret the results of classification for constructed classes. Based on the classification accuracy, the most appropriate parameters of reference signal were determined.
\end{abstract}

Keywords-reference signal, signal processing, aging, convolutional neural network

\section{INTRODUCTION}

In a modern Smart Grids, due to presence of a large amount of data, it is necessary to use algorithms for automatically determining the network status without the participation of the operator. It can be achieved by involving artificial neural networks (ANNs) in the process. In the resent years very promising cable aging state recognition methods were outlined [1], [2], [3], [4]. The paper [1] proposed, that distortion of a power line communication signals provided an information about physical properties of grid components and can be used to detect cable aging. Accordingly, variations in a channel frequency response (CFR) are used. The authors of [1] made a numerical model of a three core XLPE insulated medium voltage cable, considering the frequency range from $100 \mathrm{kHz}$ to $35 \mathrm{MHz}$. To distinguish water-treed cable model from intact cable model, a binary classification by aim of machine learning algorithm in a form of support vector machine (SVM) was utilized. The authors points out to the problem of meaningful feature selection which will be used in classifier. To overcome influence of different load conditions on classification results they used skewness and kurtosis of magnitude and phase of the normalized CFR. Work [2] likewise uses features, extracted from CFR, estimated inside a power line communication modem (PLM). Authors modelled thermal aging of a paper insulated cables using their previous measurements. In [3] was also presented supervised machine learning algorithm to extract information about grid anomalies from CFR, line impedance and reflection coefficient. In our present work, we decided to found an optimal signal for purpose of degradation levels determining. We adopted a windowed swept-sine type of a signal, that was described in [5]. For classification purposes we used a modern onedimensional convolutional neural network [6]. Feature extracting process is a part of a neural network operation for this type of network. Therefore, it is a guarantee that the most meaningful features are used for classification. Five degradation levels of cable insulation were classified simultaneously.

\section{EXPERIMENTAL SETUP}

The experimental setup consists of a personal computer (PC), FPGA development board (DB), a set of high frequency current transformers (HFCT), a signal amplifier (AMP), and a unit under test (UUT). Transmitting HFCT have an air core, both receiving HFCTs have ferromagnetic cores. Reference signal with required parameters is generated by Python program, running on the $\mathrm{PC}$. Communication with the DB is carried out using the SCPI protocol. The DB has two DA converters and two AD converters, with a resolution 14 bits and sampling frequency $125 \mathrm{MHz}$. One of the DA converters is connected to the transmitting HFCT via a $15 \mathrm{~W}$ signal amplifier with high linearity. Both $\mathrm{AD}$ converters are involved in receiving signals from receiving HFCTs. HFCT-1 (Fig. 1) is used to record the reference signal before passing through the UUT, HFCT-2 is used to record the reference signal passed through the UUT. Impedances $Z_{1}$ and $Z_{2}$ are close to zero for this particular experiment. Therefore, there occurs full reflection of both, electric and magnetic component of an incident wave from the cable ends. Time window during signal acquisition was 131072 ns. Every digitized raw signal record contains 16384 sampled values. Captured time sequences were stored on the PC for subsequent processing. As an UUT we used a coaxial cable RG59, with a dielectric made of low-density polyethylene (LDPE). The melting point of this LDPE were determined as $105,35{ }^{\circ} \mathrm{C}$ by the aim of differential scanning calorimetry analysis. To simulate different aging levels of the cable insulation we applied accelerated aging process and used physical model based on the following form of an Arrhenius equation:

$$
L_{H}=L_{0} \cdot \exp \left[\frac{E_{a}}{B_{k}}\left(\frac{1}{273+T_{c}}-\frac{1}{273+T_{a}}\right)\right]
$$

where:

$L_{H}$ - equivalent insulation age (hours)

$L_{0}$ - accelerated aging time (hours)

$E_{a}$ - activation energy of a LDPE (eV)

$B_{k}-$ Boltzmann constant $\left(8,6171 \cdot 10^{-5} \mathrm{eV} / \mathrm{K}\right)$

$T_{c}$ - operational temperature $\left({ }^{\circ} \mathrm{C}\right)$

$T_{a}$ - accelerated aging temperature $\left({ }^{\circ} \mathrm{C}\right)$ 


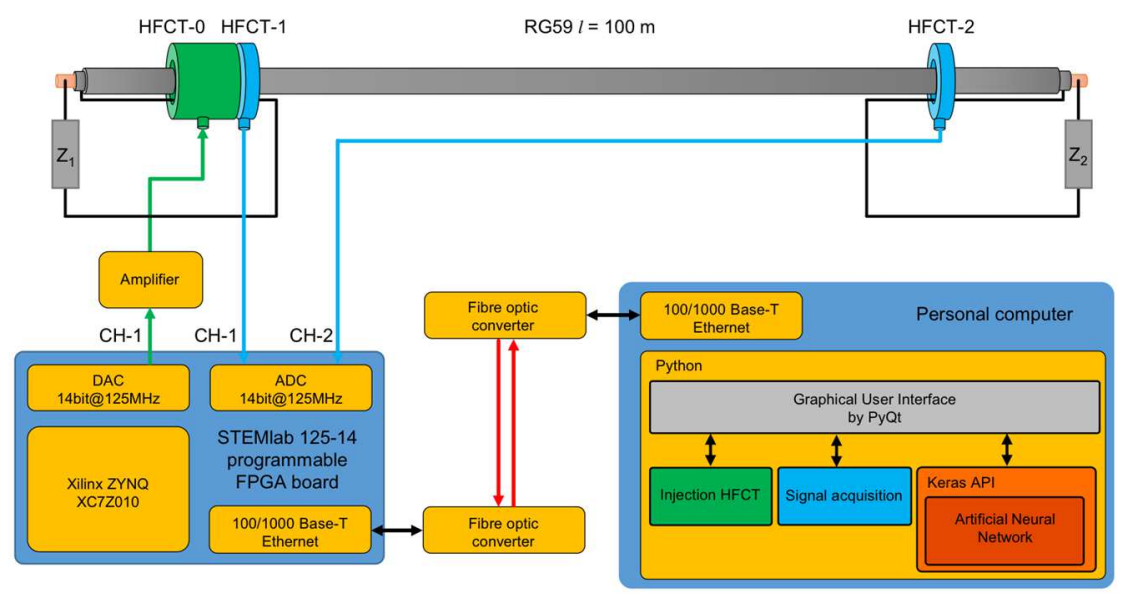

Fig. 1. Schematic representation of the experimental setup.

Accelerated aging temperature was constant during the experiment and was set to $100{ }^{\circ} \mathrm{C}$. For equivalent age calculations the operating temperature of tested cable was assumed $65{ }^{\circ} \mathrm{C}$. For the activation energy of LDPE was accepted the value $190 \mathrm{~kJ} / \mathrm{mol}$ (or 1,969 eV) [7].

\section{REFERENCE SigNAL CONSTRUCTION}

While constructing the reference signal, one must take into account many parameters to meet the conditions. General form of the reference signal can be described by the following equation:

$$
r(t)=w(t) \cdot s(t)
$$

where:

$$
\begin{aligned}
& r(t) \text { - reference signal to be send; } \\
& w(t) \text { - window; } \\
& s(t) \text { - swept-sine signal. }
\end{aligned}
$$

As a window $w(t)$ Tuckey window was applied. While choosing the minimal and maximal frequency of the sweptsine signal, the characteristics of the propagation channel properties of the UUT should be considered. Moreover, an appropriate duration of the reference signal must be chosen to avoid overlapping of the transmitted and reflected signal. Since the tested cable has a length $100 \mathrm{~m}$ and the velocity of a wave propagation is $5,2 \mathrm{~ns} / \mathrm{m}$, it was decided to set the duration of the reference signal to $1040 \mathrm{~ns}$, or 130 sampled data points. To determine an optimal reference signal shape for aging state recognition, the following steps were made. As a base reference signal, sweep frequency sinusoid was chosen. Two main groups of reference signal were constructed. The first group contains sweep frequency with a start at $1 \mathrm{MHz}$ and stop at $10 \mathrm{MHz}$, the second group contains signals with a start frequency $10 \mathrm{MHz}$ and stop frequency $1 \mathrm{MHz}$. Within each group, 9 variants were constructed using $1 \mathrm{MHz}$ step. Tukey window with the following parameters is used for signal windowing: signal length was set to 130 data points, shape parameter alpha was set to 0,3 . This parameter represents the faction of the window inside the cosine tapered region.

\section{DATA PROCESSING}

The simplified scheme of a data flow for this experiment is represented in Fig. 2. To extract the useful part from the signal, recorded by HFCT sensor, there is no need, in our case, to apply any peak detection algorithm, since the sending time of the reference signal is known in advance. We only need to

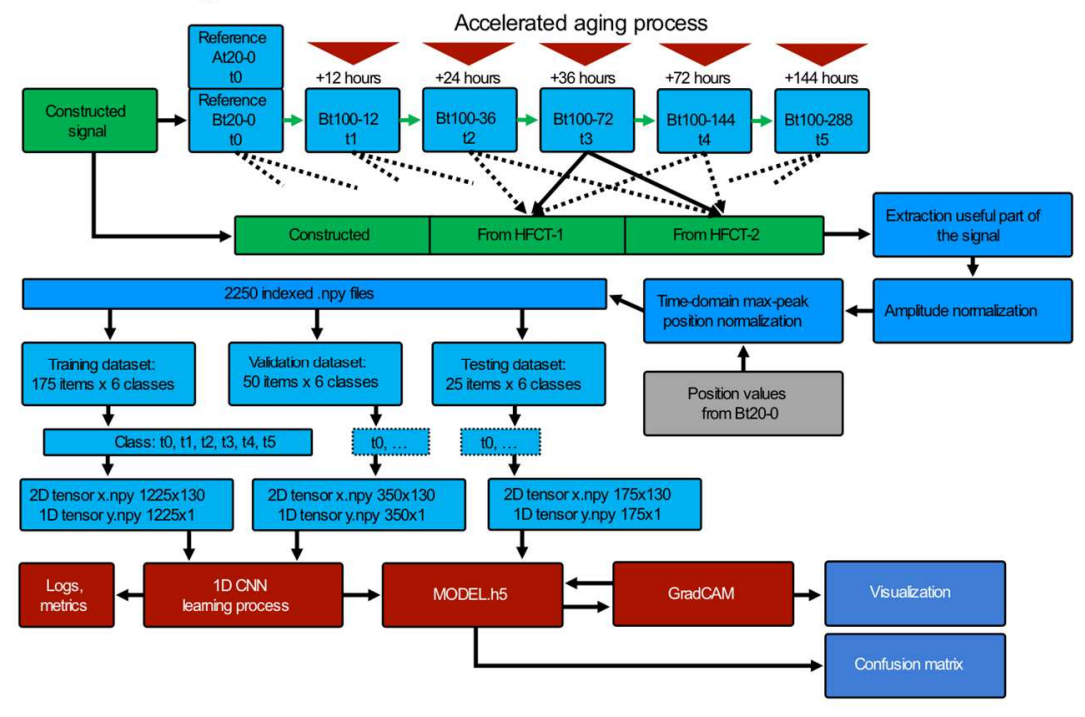

Fig. 2. Schematic representation of the data flow in the experiment. 
apply correction factors depending on the tested cable length and the propagation time of the signal through the electronic circuits of the transmitting-receiving equipment and the tested cable before extracting data from the raw signal. Since it is assumed that only subtle changes in the received reference signal will be observed, we would not like to subject the data to additional unnecessarily signal processing, thereby risking the loss of useful details. Only signal normalizations were applied. Amplitude normalization was made in the range from 0 to 1 , and time-domain normalization to max-peak position was used in the same way as described in Fig. 3. Since the network can learn to distinguish between classes based only on the whole signal shift, it is necessary to exclude this possibility. For these purposes max-peak position normalization is used.

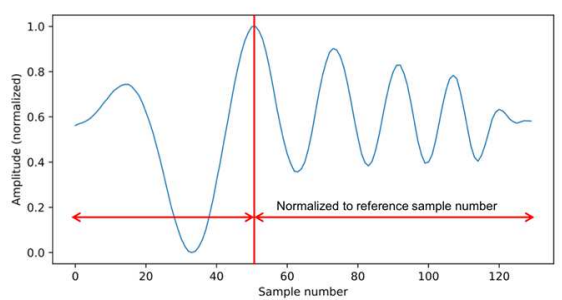

Fig. 3. To explaining time-domain max-peak position normalization.

\section{Classes Definition}

In total, 6 classes were defined from data obtained from the accelerated aging experiments. Five classes were constructed for measuring on the Cable B, while Cable A was used as a reference. The names of the relative classes, described in the Tab. 1, reflects the cable name (A, B), applied temperature $\left(\mathrm{t} 20-20^{\circ} \mathrm{C}, \mathrm{t} 100-100{ }^{\circ} \mathrm{C}\right)$ and duration of accelerated ageing process $(0,12,36,72$ and 144 hours).

TABLE I. CABLE PARAMETERS FOR DIFFERENT ACCELERATED AGING DURATION AND CORRESPONDING ClASS NAMES

\begin{tabular}{|l|c|c|c|c|c|}
\hline \multicolumn{1}{|c|}{ Name } & $\begin{array}{c}\text { Class } \\
\text { name }\end{array}$ & $\begin{array}{c}\text { Cumulative } \\
\text { acceleration } \\
\text { aging } \\
\text { duration } \\
\text { (hours) }\end{array}$ & $\begin{array}{c}\text { Equivalent } \\
\text { age by } \\
\text { Arrhenius } \\
\text { equation } \\
\text { (years) }\end{array}$ & $\begin{array}{c}\mathrm{C} \\
(\mathrm{pF} / \mathrm{m})\end{array}$ & $\begin{array}{c}\mathrm{R}_{\mathrm{DC}} \\
(\Omega / \mathrm{m})\end{array}$ \\
\hline A-t20-0 & - & 0 & 0 & 70,07 & $60,6 \cdot 10^{-3}$ \\
\hline B-t20-0 & $\mathrm{t} 0$ & 0 & 0 & 70,07 & $60,6 \cdot 10^{-3}$ \\
\hline B-t100-12 & $\mathrm{t} 1$ & 12 & 0,8 & 68,50 & $60,0 \cdot 10^{-3}$ \\
\hline B-t100-36 & $\mathrm{t} 2$ & 36 & 2,3 & 68,21 & $63,3 \cdot 10^{-3}$ \\
\hline B-t100-72 & $\mathrm{t} 3$ & 72 & 4,7 & 68,69 & $59,8 \cdot 10^{-3}$ \\
\hline B-t100-144 & $\mathrm{t} 4$ & 144 & 9,3 & 69,07 & $59,4 \cdot 10^{-3}$ \\
\hline
\end{tabular}

Deep convolutional neural network is able to find meaningful features in the training data set without manual feature engineering. This can be achieved with a sufficient number of training examples, which can be obtained by data augmentation. In our experiment we use physical data augmentation as opposed to mathematical. The reason is that mathematical augmentation cannot produce new information but only remixes existing. Physical data augmentation in this experiment occurs at the stage of data acquisition via changing the parameters of the physical interaction between the sensors and the object under test. The principle of the physical data augmentation is described in Tab. 2. Augmentation was achieved by changing a position of transmitting and receiving HFCTs for every data set according to the scheme introduced in the Tab 2. It can be seen that every data set was augmented by factor 25 , since HFCT-0 has 5 different positions, and HFCT -2 also. For determining the aging state of a UUT was used already defined construction of the one-dimensional convolutional ANN, presented in our earlier work [6], with a few parameters tuned. The network consists of two one dimensional convolutional layers, which are used for feature extraction, and two dense layers for classification task. The first layer of network have 20 filters with a kernel size 51; the second layer have 60 filters with a kernel size 3. Used activation function is ReLU, optimizer - Adam.

TABLE II. The Principle of The Physical Data AugMENTATION

\begin{tabular}{|c|c|c|c|c|}
\hline \multirow{2}{*}{$\begin{array}{l}\text { Variant } \\
\text { number }\end{array}$} & \multicolumn{4}{|c|}{ HFCTs placement variants } \\
\hline & \multicolumn{2}{|c|}{ HFCT-1 } & \multicolumn{2}{|c|}{ HFCT-2 } \\
\hline 1 & \multirow{5}{*}{ position 0} & \multirow{5}{*}{$0 \mathrm{~cm}$} & position 0 & $0 \mathrm{~cm}$ \\
\hline 2 & & & position 1 & $5 \mathrm{~cm}$ \\
\hline 3 & & & position 2 & $10 \mathrm{~cm}$ \\
\hline 4 & & & position 3 & $15 \mathrm{~cm}$ \\
\hline 5 & & & position 4 & $20 \mathrm{~cm}$ \\
\hline 6 & \multirow{5}{*}{ position 1} & \multirow{5}{*}{$5 \mathrm{~cm}$} & position 0 & $0 \mathrm{~cm}$ \\
\hline 7 & & & position 1 & $5 \mathrm{~cm}$ \\
\hline 8 & & & position 2 & $10 \mathrm{~cm}$ \\
\hline 9 & & & position 3 & $15 \mathrm{~cm}$ \\
\hline 10 & & & position 4 & $20 \mathrm{~cm}$ \\
\hline \multicolumn{3}{|c|}{ The same permutations for positions $2,3,4$} & position 0 & $0 \mathrm{~cm}$ \\
\hline$\ldots$ & \multirow{3}{*}{$\begin{array}{l}\text { position } 2 \\
\text { position } 3 \\
\text { position } 4\end{array}$} & \multirow{3}{*}{$\begin{array}{l}10 \mathrm{~cm} \\
15 \mathrm{~cm} \\
20 \mathrm{~cm}\end{array}$} & $\begin{array}{l}\text { position } 1 \\
\text { position } 2\end{array}$ & $\begin{array}{r}5 \mathrm{~cm} \\
10 \mathrm{~cm}\end{array}$ \\
\hline 24 & & & position 3 & $15 \mathrm{~cm}$ \\
\hline 25 & & & position 4 & $20 \mathrm{~cm}$ \\
\hline
\end{tabular}

\section{Classification AND Results}

Network learning was conducted on GPU. For applying a classification algorithm, the received and processed HFCT-2 signals were divided in the following way: 175 records within every class were used as a training data set, 50 records were used as a validation data set and 25 records as a testing data set. Classification results, for the case when only amplitude

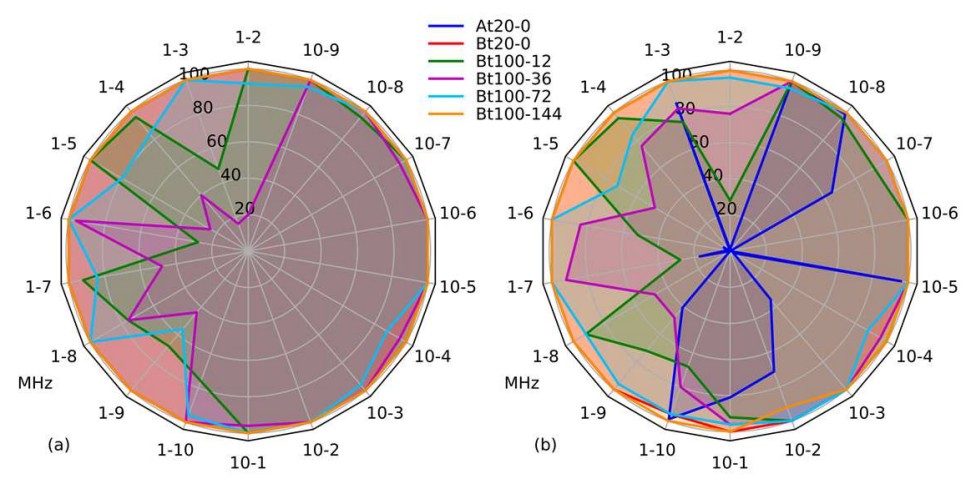

Fig. 4. Classification accuracy of an aging state of the cable, depending on reference signal frequencies. 


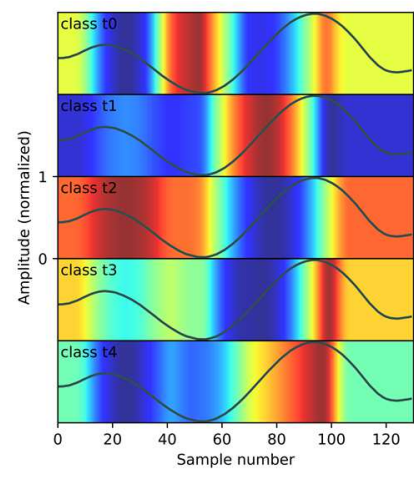

$1-2 \mathrm{MHz}$

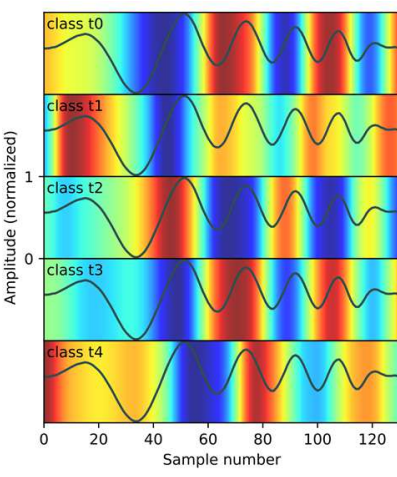

$1-10 \mathrm{MHz}$

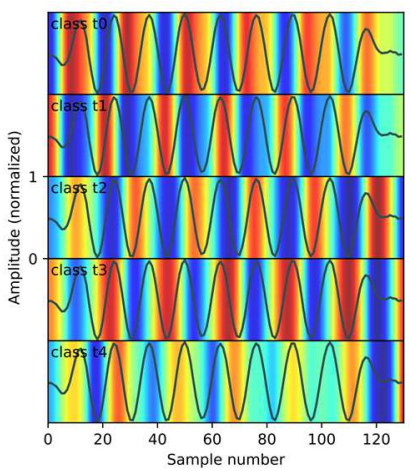

$10-9 \mathrm{MHz}$

Fig. 5. Example of an application of Grad-CAM [8] visualization method for selected reference signal frequencies.

normalization of the signal was applied are represented in Fig. 3(a). For this case is observable very high rate of correct results for reference signals with sweep frequency variants from $10-1 \mathrm{MHz}$ to $10-9 \mathrm{MHz}$. Classification accuracy was 96-100\%, except for the reference signal with the frequency sweep 10-4 MHz, where the classification accuracy for class B-t100-72 was only $88 \%$. For low reference signal frequencies (from 1-2 MHz to $1-10 \mathrm{MHz}$ ) there is observable low classification accuracy for the classes B-t100-12, B$\mathrm{t} 100-36$ and $\mathrm{B}-\mathrm{t} 100-72$, while for the classes B-t20-0 and Bt100-144 the classification accuracy was $100 \%$ for all used frequencies. Furthermore, all 250 records from class A-t20-0 were used as a testing data set, while only records from cable $\mathrm{B}$ were used for network learning process. As a result, for all 18 reference signal variants were obtained $100 \%$ accuracy. In Fig. 3(b) are represented the results of aging state classification for the case when time-domain max-peak normalization was applied during data processing. This time, influence of the whole signal shifting on the learning process of the ANN was excluded. Parity remains almost the same with minor changes and with a tendency of the classification accuracy to be higher for higher frequencies. Radical changes have occurred in classification accuracy when the data from variant $\mathrm{A}-\mathrm{t} 20-0$ were used as the testing data set. Now only the reference signal frequencies $1-3 \mathrm{MHz}, 1-10 \mathrm{MHz}, 10-5$ $\mathrm{MHz}, 10-8 \mathrm{MHz}$ and $10-9 \mathrm{MHz}$ are high enough to make the final decision on which class the data belongs to. Low classification accuracy for low reference signal frequencies explains Fig. 4. Red areas on the signal represents ANN "attention", in other words, using this areas, the neural network distinguishes one class from another. And for the signals with higher frequencies, there are more possibilities for ANN to find useful features in the signal changes.

\section{CONCLUSIONS}

From the experiment it can be conclude, that it is possible to use as a reference signal swept-sine signal with the length $1040 \mathrm{~ns}$ and consisting 130 data points, to determine thermal degradation level of insulation of coaxial cable by aim of one- dimensional convolutional neural network. For data augmentation was used method, when a position of receiving sensor is changed during acquisition. According to classification results, its method is very promising for data augmentation. To normalize a signal in a time domain, method of maximum signal peak amplitude was applied. To visualize learned by the network features, Grad-CAM visualization method was successfully applied.

\section{ACKNOWLEDGMENT}

This work was supported by the student research project SGS-2021-018.

\section{REFERENCES}

[1] L. Forstel and L. Lampe, "Grid diagnostics: Monitoring cable aging using power line transmission," 2017 IEEE International Symposium on Power Line Communications and its Applications (ISPLC), 2017.

[2] F. Yang, W. Ding, and J. Song, "Non-intrusive power line quality monitoring based on power line communications," 2013 IEEE 17th International Symposium on Power Line Communications and Its Applications, 2013.

[3] Y. Huo, G. Prasad, L. Atanackovic, L. Lampe, and V. C. M. Leung, "Cable Diagnostics With Power Line Modems for Smart Grid Monitoring," IEEE Access, vol. 7, pp. 60206-60220, 2019.

[4] N. A. Letizia and A. M. Tonello, "Supervised Fault Detection in Energy Grids Measuring Electrical Quantities in the PLC Band," 2020 IEEE International Symposium on Power Line Communications and its Applications (ISPLC), 2020.

[5] J. Wang, P. Stone, Y.-J. Shin, and R. Dougal, "Application of joint time-frequency domain reflectometry for electric power cable diagnostics," IET Signal Processing, vol. 4, no. 4, p. 395, 2010.

[6] M. Olkhovskiy, E. Müllerová, and P. Martínek, "Impulse signals classification using one-dimensional convolutional neural network," Journal of Electrical Engineering, vol. 71, no. 6, pp. 397-405, 2020.

[7] J. W. Park, S. C. Oh, H. P. Lee, H. T. Kim, and K. O. Yoo, "Kinetic analysis of thermal decomposition of polymer using a dynamic model," Korean Journal of Chemical Engineering, vol. 17, no. 5, pp. 489-496, 2000.

[8] R. R. Selvaraju, M. Cogswell, A. Das, R. Vedantam, D. Parikh, and D. Batra, "Grad-CAM: Visual Explanations from Deep Networks via Gradient-Based Localization," 2017 IEEE International Conference on Computer Vision (ICCV), 2017. 